\title{
ZenoFishDb v1.1: A Database for Xenotransplantation Studies in Zebrafish
}

\author{
Seniye Targen, ${ }^{1}$ Tuğberk Kaya, ${ }^{2,3}$ M. Ender Avci, ${ }^{4}$ Damla Gunes, ${ }^{2}$ Ayse Gokce Keskus, ${ }^{2}$ and Ozlen Konu ${ }^{1,2,5}$
}

\begin{abstract}
Rapidly accumulating literature has proven feasibility of the zebrafish xenograft models in cancer research. Nevertheless, online databases for searching the current zebrafish xenograft literature are in great demand. Herein, we have developed a manually curated database, called ZenoFishDb v1.1 (https://konulab.shinyapps.io/ zenofishdb), based on R Shiny platform aiming to provide searchable information on ever increasing collection of zebrafish studies for cancer cell line transplantation and patient-derived xenografts (PDXs). ZenoFishDb v1.1 user interface contains four modules: DataTable, Visualization, PDX Details, and PDX Charts. The DataTable and Visualization pages represent xenograft study details, including injected cell lines, PDX injections, molecular modifications of cell lines, zebrafish strains, as well as technical aspects of the xenotransplantation procedures in table, bar, and/or pie chart formats. The PDX Details module provides comprehensive information on the patient details in table format and can be searched and visualized. Overall, ZenoFishDb v1.1 enables researchers to effectively search, list, and visualize different technical and biological attributes of zebrafish xenotransplantation studies particularly focusing on the new trends that make use of reporters, RNA interference, overexpression, or mutant gene constructs of transplanted cancer cells, stem cells, and PDXs, as well as distinguished host modifications.
\end{abstract}

Keywords: zebrafish, xenograft, cancer, database, R shiny, patient-derived xenograft

\section{Introduction}

$\mathbf{T}$ UMOR XENOGRAFT MODELS, particularly of rodents, have long been used in scientific research. ${ }^{1-4}$ Today's state-ofthe-art technologies allow use of transgenic rodent models in cancer research through cell line-derived xenotransplantation $^{5}$ and transplantation of patient-derived xenografts (PDXs). ${ }^{5,6}$ Innumerable xenograft studies performed in rodents have resulted in great demand for established bibliotheca where information from them could be entered and updated collectively providing easy access. Accordingly, several databases or tools exhibiting collection of rodent xenotransplantation studies have been developed, and they mainly focus on PDX studies in mouse models. ${ }^{7-10}$ For example, MTB (Mouse Tumor Biology) ${ }^{7}$ provides information on tumor, strain, genetic architecture, pathology images, and gene expression datasets, as well as providing a link to The Jackson Laboratory and EMBL-EBI joint project, PDX
Finder. ${ }^{11}$ In addition, organ specific xenograft databases of mouse models are also present, ${ }^{9}$ while a commercial xenograft cell line database by Taconic Biosciences, Inc., ${ }^{12}$ provides another platform for cell-line specific transplantations.

Zebrafish is a valuable vertebrate model organism that has more recently emerged in the xenograft field. ${ }^{13}$ The use of zebrafish embryos in xenotransplantation has generated novel avenues for researchers to explore different aspects of basic and applied sciences, including cancer biology as reviewed in the literature. ${ }^{14-16}$ Moreover, xenograft studies in zebrafish offer enormous benefits and a broad range of applications since effects of transient or stable modifications in immortalized or primary cell lines can be tested during embryogenesis/organogenesis. In particular, the modifications introduced by overexpression vectors, ${ }^{17-19}$ as well as RNA interference technologies, ${ }^{20,21}$ help identify gene- and/or mutation-specific effects on tumor characteristics in vivo in zebrafish. However, the increasing number of zebrafish

\footnotetext{
${ }^{1}$ Department of Molecular Biology and Genetics, Bilkent University, Ankara, Turkey.

${ }^{2}$ Interdisciplinary Program in Neuroscience, Bilkent University, Ankara, Turkey.

${ }^{3}$ Institute of Neuronal Cell Biology, Technical University Munich, Munich, Germany.

${ }^{4}$ Izmir Biomedicine and Genome Center, Dokuz Eylul University, Izmir, Turkey.

${ }^{5}$ UNAM-Institute of Materials Science and Nanotechnology, Bilkent University, Ankara, Turkey.
} 
xenograft studies in cancer biology has made systematic analysis and curation necessary.

Herein, first ever zebrafish-specific xenograft database, ZenoFishDb v1.1, has been generated using Shiny package ${ }^{22}$ in the $\mathrm{R}$ programming environment ${ }^{23}$ with a particular focus on zebrafish transplantation studies of molecularly modified cells, PDXs, and cancer stem cells (CSCs), as well as those performed on modified hosts.

\section{Materials and Methods}

\section{Contents of ZenoFishDb v1.1}

We have reviewed and manually curated the literature regarding zebrafish xenograft studies, particularly focusing on molecular- and strain-specific modifications; and an updatable excel spread sheet containing different attributes from the selected studies has been generated. Accordingly, the data used in ZenoFishDb v1.1 include different individual research elements/fields extracted from full texts, including the type of cancer, injected cell line or cell type, taxonomic species of the injected cell line, type of the molecular modification (e.g., overexpression, short hairpin RNA [shRNA], small interfering RNA [siRNA]), official name of the modified gene, number of cells injected, injection site and time, developmental stage of the fish, name of the injected zebrafish line, fluorescence source (or reporter), biological assessment (e.g., invasion, angiogenesis, tumor size), type of host strain modifications (e.g., transgenes and mutations), and references, including PubMed IDs. The excel spread sheet has been imported into the $\mathrm{R}$ environment before parsing and processing for downstream analyses and visualization processes.

\section{Development of ZenoFishDb v1.1 using R Shiny}

ZenoFishDb v1.1 is an interactive web application developed using the Shiny framework in R. ${ }^{22,23}$ The database features four main components: DataTable, Visualization, PDX Details, and PDX Charts.

The DataTable provides sorting, pagination, and filtering while containing comprehensive information about xenograft studies in ZenoFishDb v1.1 using the DT package, an R interface of JavaScript library DataTables. ${ }^{24}$ In addition to the intrinsic filtering operations done by DataTables library, other filtering options are presented to the user upon selection of attributes of interest and respective subselections based on dplyr package. ${ }^{25}$

ZenoFishDb v1.1 Visualization page allows for the statistical analysis of selected data. This component of the database operations works upon selection of a column of interest from the uploaded excel file to display pie and/or bar chart of the proportional distribution of the selected data using Plotly, an open source R graphing library. ${ }^{26}$

PDX Details and PDX Charts utilize the same R packages for tabular data manipulation and visualization as the previously aforementioned components of the application, while expanding on the PDX study details specifically. ZenoFishDb v1.1 is hosted and maintained online at shinyapps.io servers. Updates are planned biannually and will be performed upon collection and manual curation of new publications as they arise in the zebrafish xenograft research field.

\section{Results}

ZenoFishDb v1.1: DataTable, Visualization, PDX Details, PDX chart modules

ZenoFishDb v1.1 enables a thorough search for existing zebrafish xenograft studies in the literature focusing on those with molecular interventions and/or involving use of stem cells and PDXs. With this intention, the literature has been mined for "zebrafish xenograft," "zebrafish xenotransplant," “zebrafish xenotransplantation," “zebrafish patient derived xenograft," "zebrafish xenograft microenvironment," “zebrafish xenograft morpholino," "zebrafish xenograft crispr," “zebrafish xenograft mutation," “zebrafish xenograft primary cell," and similar keywords through NCBI PubMed search page. A total number of 211 studies focusing on the application of molecularly modified cell, PDX, and/or stem cell transplantations, as well as studies with distinct host modifications and microenvironments, have been incorporated into the current version of ZenoFishDb v1.1 manually. Accordingly, the reviewed literature and curated data have been projected onto four compartments and described in detail as follows.

The DataTable provides information on the technical and biological details of research articles in a table format. The origin of transplanted cancer cells and/or tissue, their abbreviations, species of the injected cell lines, injected cell lines and cell lines subjected to molecular modifications, modified genes, available PDX studies, stem cell properties of injected cells, treatments applied to xenografts, injection sites, original and categorized injected cell numbers, developmental stage, injection time, zebrafish strains, host modifications and their details, cell tracking sources, biological assessments, tumor assessment end points, references, and PubMed hyperlinks are included in the DataTable. A finetuned search is also available through the "Attributes" and the "Subselections" tabs on the DataTable (Fig. 1A).

The Visualization webpage is designed to deliver graphical and statistical data for the information displayed through the DataTable. Herein, an attribute could be selected through the "Columns" tab, and the schematic representation could be accessed through the "Bar Chart" and "Pie Chart" options. The information provided through the page includes the number of total variables, unique variables, and percentage of the selected attribute. Visualization and generation of figures can be manually adjusted through "Chart height," "Legend font size," "Inside text font size," and "Barplot label size", options, and images can be downloaded as .png files. In addition, information represented on histograms can be downloaded in the table format. A screenshot displaying all the features of the Visualization module has been provided with an example attribute, that is, "cancer/tissue of origin" (Fig. 1B and Supplementary Table S1).

The PDX Details module (Fig. 2A) is designated to deliver cumulative information on the PDX studies incorporated to ZenoFishDb v1.1. Herein, the data on patients and/or tumors, including age/sex/ethnicity, disease name, primary site, metastasis or recurrence status, treatment status, clinical information, cytogenetic information, karyotype analysis, and other relevant data, are provided. In addition, details about the engraftment have also been incorporated for each case. These features include type of injection (patient-derived tissue engraftment [PDX-tissue] or tissue-derived cell line engraftment 

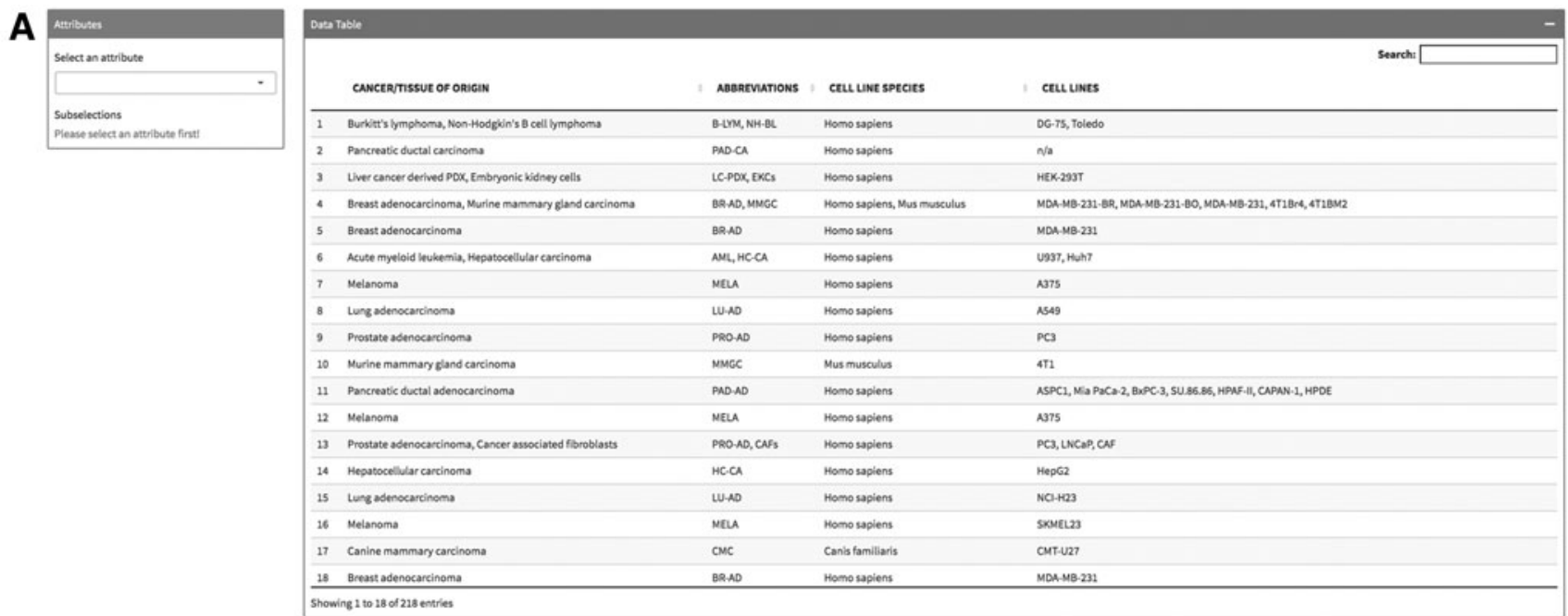

B
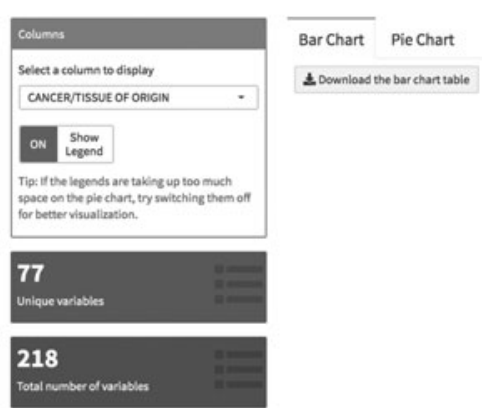

A Downlosd the bar chan ente
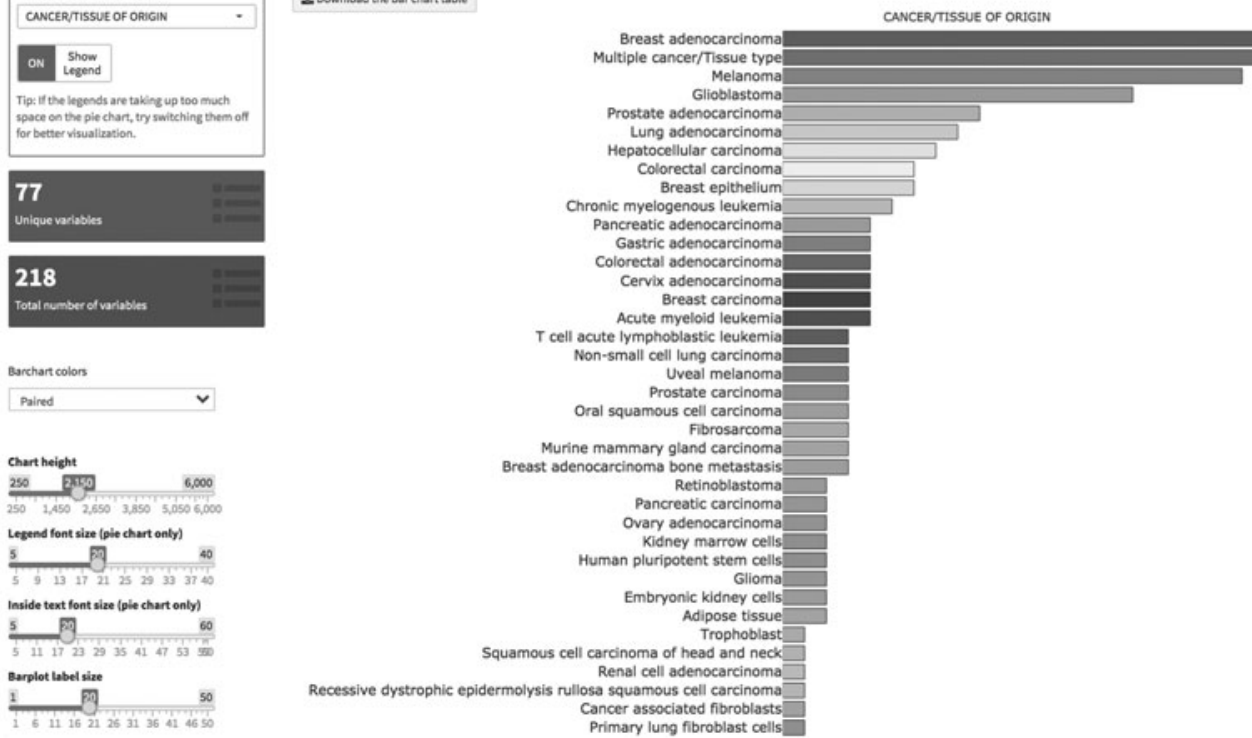

FIG. 1. DataTable and Visualization modules of ZenoFishDb v1.1. (A) Screenshot of DataTable displaying the list of the reviewed and manually curated data in a table format. Selected articles are displayed in descending order according to their release dates as default. Selection and subselection tabs enable fine-tuned search categories providing detailed information for the selected items. (B) Screenshot of introductory Visualization page displaying the overview of this module with an example of descriptive statistics of the cancer types/tissue of origin entitled with full names. Figures are available in greater detail online.

[PDX-cell line]), injected cell numbers, injection site, fish strain, and injection period together with relevant PMID ID.

The PDX Charts module (Fig. 2B) has been integrated to display the bar chart of the most frequently mentioned attributes of the PDX Details module. These attributes include detailed nature of disease, sex, primary/metastatic/recurrent status, zebrafish line, injection period, PDX-cell line/PDXtissue information, and cell numbers.

\section{Types of cancers studied using zebrafish xenograft models}

The feasibility of transplantation of immortalized cells, PDXs, primary cells or stem cells into zebrafish embryos, juvenile, ${ }^{27}$ and/or adult fish ${ }^{28}$ offers an exquisite opportunity for assessing various aspects of tumor biology. ${ }^{29-36}$ Searching the current version of ZenoFishDb v1.1, we have identified that breast adenocarcinoma $(14.74 \%)$ is the most studied cancer followed by multiple cancer/tissue types (MULTIPLE) (10.76\%), melanoma $(8.37 \%)$, and glioblastoma (6.38\%) (Fig. 1B and Supplementary Table S1). Expectedly, a cell line of breast adenocarcinoma origin, MDA-MB-231 (8.71\%), accounts for the most investigated cell line, whereas majority of the cancer types or tissue of origins are represented by a single cell line (Supplementary Table S2). The injected cell lines belong to human (80.51\%), mouse $(14.41 \%)$, zebrafish $(2.97 \%)$, rat $(1.27 \%)$, goldfish $(0.42 \%)$, and $\operatorname{dog}(0.42 \%)$. 


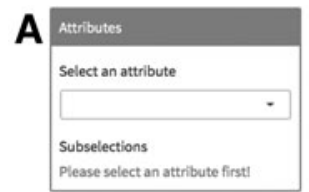

\begin{tabular}{|c|c|c|c|c|c|c|c|}
\hline $\operatorname{poxc}$ & etealis & & & & & & - \\
\hline & & & & & & Search: $\square$ & \\
\hline & Mentioned PDX-ID/NAME: & $\begin{array}{l}\text { ZenofishDb } \\
\text { PDX-1D: }\end{array}$ & 1) Age: ! & Sex: 1 & Ethnicity: : & Detailed nature of disease: & 3 \\
\hline 1 & GBM9 & ZFDB_31031007.1 & 41 & Male & $\mathrm{n} / \mathrm{a}$ & Cortical glioblastoma & \\
\hline 2 & SJRHB000026,X1 & ZFOB_31031007.2 & 4 & Female & $\mathrm{n} / \mathrm{a}$ & Embryonal Rhabdomyosarcoma & \\
\hline 3 & SJRHBO12 $Y$ & ZFOB_310310073 & 17 & Male & $\mathrm{n} / \mathrm{a}$ & Embryonal Rhabdomyosarcoma & \\
\hline 4 & MELI67C & ZFOB_31031007.4 & 49 & Male & $n / a$ & Melanoma & \\
\hline 5 & MEL268C & ZFOB_31031007.5 & $n / a$ & Male & $\mathrm{n} / \mathrm{a}$ & Melanoma & \\
\hline 6 & $B R x-07$ & ZFDB_31031007.6 & $n / a$ & Female & $n / a$ & Breast cancer & \\
\hline 7 & PDACH1127 & ZFOB_31107449.1. & n/a & $n / a$ & n/a & Pancreatic ductal adenocarcinoma & \\
\hline 8 & PDACHO827 & ZFOB_31107449.2.2 & $n / a$ & $n / a$ & $\mathrm{n} / \mathrm{a}$ & Pancreatic ductal adenocarcinoma & \\
\hline 9 & PDACNOB31 & ZFDB_31107449.3.3 & $\mathrm{n} / \mathbf{a}$ & $n / \mathbf{a}$ & $\mathrm{n} / \mathrm{a}$ & Pancreatic ductal adenocarcinoma & \\
\hline 10 & Patient 1 (P1-Parental) & ZFDB_31164413 & 81 & Female & White & Pancreatic ductal adenocarcinoma & \\
\hline 11 & $\operatorname{ACC} 29$ & ZFDB_31397841 & 51 & Female & $\mathrm{n} / \mathrm{a}$ & Primary Adrenocortical carcinoma (ACC) & \\
\hline 12 & L/2 & 2FDB_31141996.1 & $n / a$ & $n / a$ & $\mathrm{n} / \mathrm{a}$ & Liver cancer & \\
\hline 13 & LaA & ZFDB_31141996.2 & $n / a$ & $n / a$ & n/a & Liver cancer & \\
\hline 14 & Las & 2FDB_31141996.3 & $n / \mathbf{a}$ & $n / a$ & $\mathrm{n} / \mathrm{a}$ & Liver cancer & \\
\hline 15 & La6 & ZFOB__31141996.4 & $\mathrm{n} / \mathbf{a}$ & $n / a$ & $\mathrm{n} / \mathrm{a}$ & Liver cancer & \\
\hline 16 & L.7 & ZFOB__31141996.5 & $n / a$ & $n / a$ & n/a & Liver cancer & \\
\hline 17 & Lo8 & ZFOB_31141996.6 & n/a & $n / a$ & n/a & Liver cancer & \\
\hline 18 & La9 & ZFDB__31141996.7 & $\mathrm{n} / \mathrm{a}$ & $\mathrm{n} / \mathrm{a}$ & $n / a$ & Liver cancer & \\
\hline
\end{tabular}

B
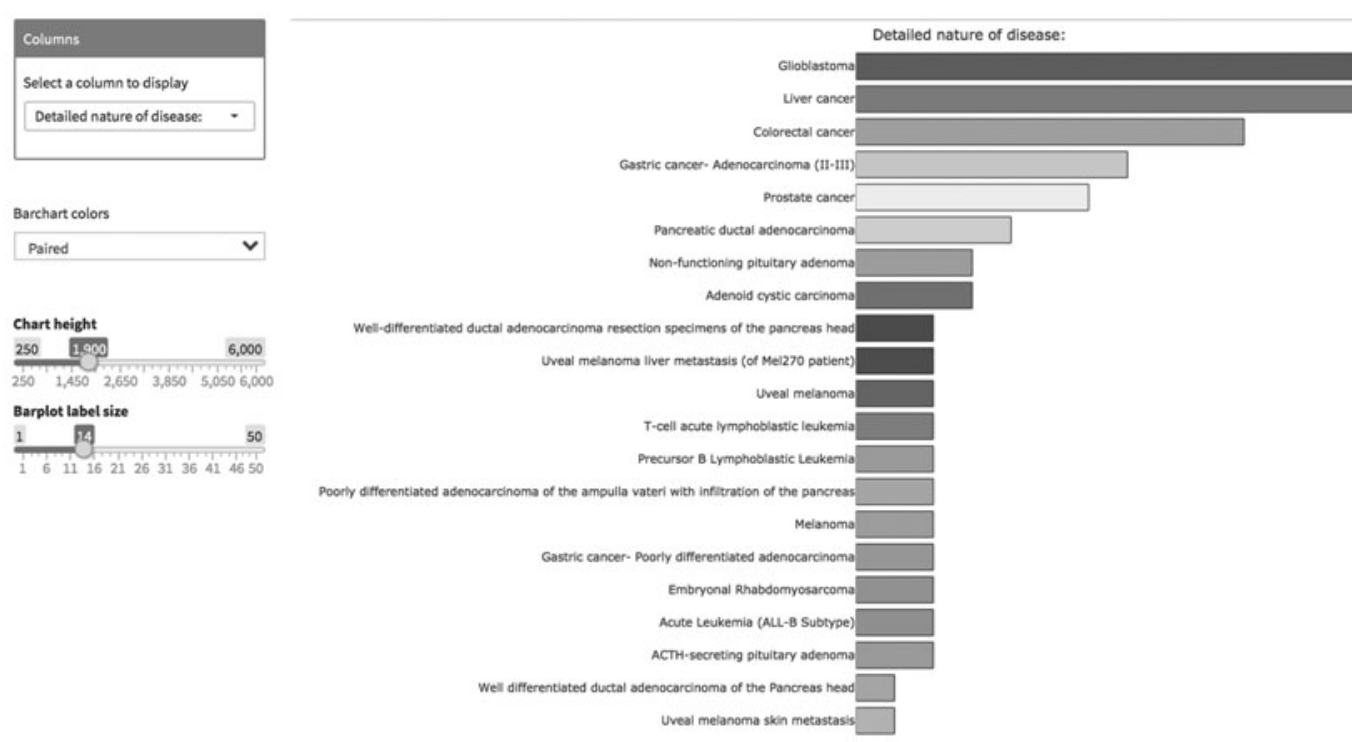

FIG. 2. $P D X$ Details and PDX Charts modules of ZenoFishDb v1.1. (A) Screenshot of the PDX Details page displaying patient details and detailed nature of disease. (B) Screenshot of the PDX Charts displaying the graphical representation for detailed disease nature of the transplanted tissue or primary cell line. PDX, patient-derived xenograft. Figures are available in greater detail online.

\section{The nature of zebrafish xenografts: molecularly modified cells, PDXs, and stem cells}

ZenoFishDb v1.1 prioritizes the molecularly modified cell transplantations that have been useful for establishing gene functionality in tumorigenesis ${ }^{37,38}$ and associated events such as proliferation, ${ }^{39,40}$ invasion, ${ }^{41,42}$ angiogenesis, ${ }^{43,44}$ metastasis, ${ }^{18,45}$ apoptosis, ${ }^{36}$ and cytotoxicity. ${ }^{46}$ Our thoroughly systematized data curation emphasizes the molecular modifications (e.g., cells accommodating transient and/or stable overexpression vectors, ${ }^{17,47,48}$ interfering RNAs $^{41,49}$ and/or Crispr-Cas9/TALEN/ZFN/Cre-LoxP ${ }^{44,50,51}$ technologies) performed in cells used for transplantation.

A ZenoFishDb v1.1 search shows that these molecular modifications predominantly include siRNA $(9.81 \%)$, shRNA
(10.94\%), expression vectors (12.45\%), CRISPR/Cas9 (0.38\%), and tag expression vectors $(37.36 \%)$ for tracking purposes (Fig. 3A and Supplementary Table S3). In addition, the cell lines subjected to molecular modifications have been also separately attributed as "modified cell lines" and can be displayed through the Visualization page and are now provided in the table format (Supplementary Table S4). Among different molecularlymodified cell lines, MDA-MB-231 (7.19\%), MCF7 (2.40\%), U-87MG (2.74\%), and PDXs (2.06\%) represent the commonly modified cells in zebrafish xenograft studies incorporated into our database.

Another highlight of ZenoFishDb v1.1 is the inclusion of PDXs along with their clinical and genetic details when available. Patient-derived xenografting is achieved through direct transplantation of patient derived tissues ${ }^{52}$ or primary 
A
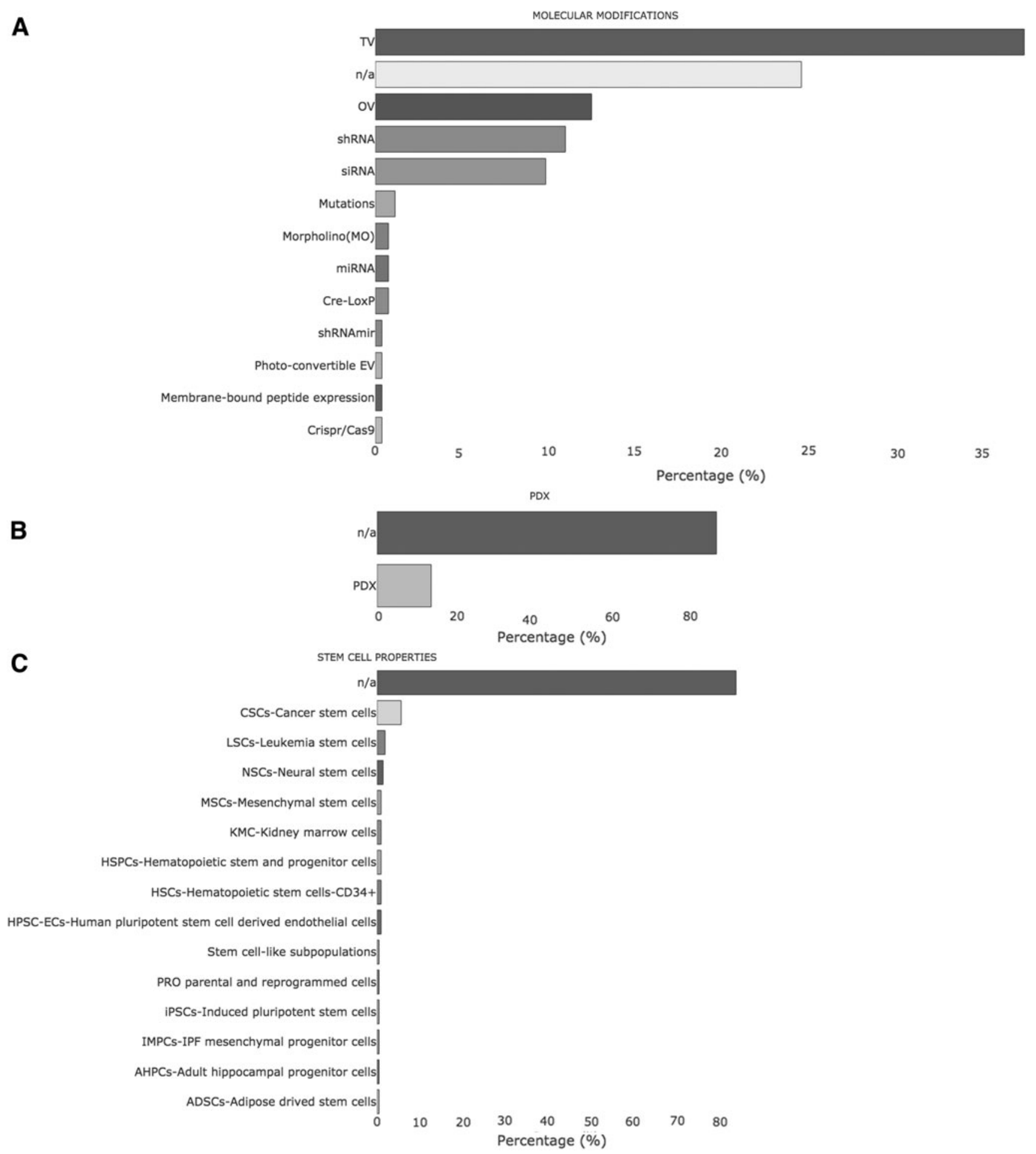

FIG. 3. The nature of xenograft studies represented on the ZenoFishDb v1.1: Molecular modifications, modified cell lines, PDXs, and stem cells. (A) Molecular modifications; (B) PDXs; (C) stem and cancer stem cell studies. Figures are available in greater detail online.

cell cultures with minimal passage numbers ${ }^{53}$ and is ideal for mirroring the true nature of carcinogenesis. In fact, implantation of PDXs from cancerous tissues in comparison to immortalized cell lines better represents patient's genomic status and the tumor heterogeneity. ${ }^{54,55}$ Altogether, the advantageous features of PDXs allow drug screening and development of personalized therapy both in rodents and zebrafish. ${ }^{55-57}$ Hence, zebrafish PDX models have also been incorporated into ZenoFishDb v1.1 through PubMed search using a keyword query of "zebrafish patient derived xenograft" or "zebrafish xenograft primary cells" keywords. This has revealed the various types of cancers used in such studies, including breast cancer bone metastasis, ${ }^{58}$ colorectal cancer, ${ }^{59}$ multiple myeloma, ${ }^{34} \mathrm{~T}$ cell acute lymphoblastic 
leukemia, ${ }^{60}$ gastric cancer, ${ }^{35}$ neuroendocrine tumors, ${ }^{61}$ adenoid cystic carcinoma, ${ }^{33}$ glioblastoma, ${ }^{62}$ as well as primary cells/tissues. ${ }^{63}$ Curated PDX studies represent $13.74 \%$ of the studies incorporated into ZenoFishDb v1.1 (Fig. 3B).

Current version of the database also houses in-detail information on the PDXs accessible through the PDX Details and PDX Charts pages as explained above (Fig. 2A, B). Most frequently provided elements/attributes of the PDX details hence can be analyzed through PDX Charts. For instance, the number of glioblastoma patients recorded accounts for the highest number/percentage followed by the liver and colorectal cancer patients among many others, including prostate cancer, pancreatic ductal adenocarcinoma, melanoma, and

A

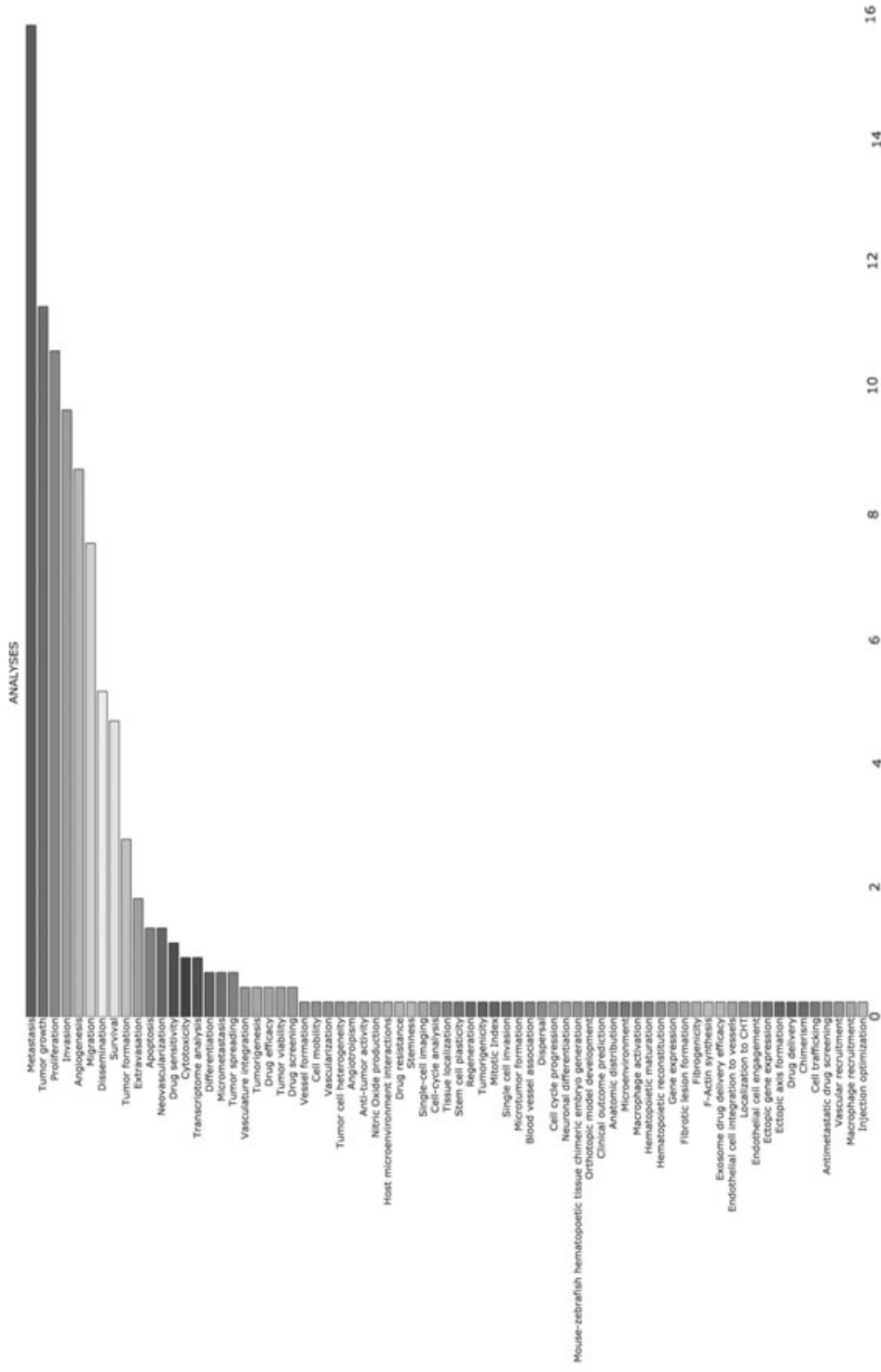

acute leukemia (Fig. 2B). ZenoFishDb v1.1, therefore, is the first database accommodating detailed and searchable information from PDX studies in the zebrafish model.

ZenoFishDb v1.1 also houses the xenograft studies using stem cells (SCs) obtained from normal tissue or cancer tissue of origin. Xenografting of CSCs of blood cancers ${ }^{64}$ and solid tumors of different origins ${ }^{65}$ to rodents has paved the way for understanding behavior of CSCs in cancer development and therapy assessments. Zebrafish model organism serving as host for CSC transplantation also enables, for example, the assessment of metastatic behavior and drug screening in prostate cancer, ${ }^{66}$ migratory behavior in breast cancer, ${ }^{67}$ and proliferative behavior in leukemia stem cells. ${ }^{68}$

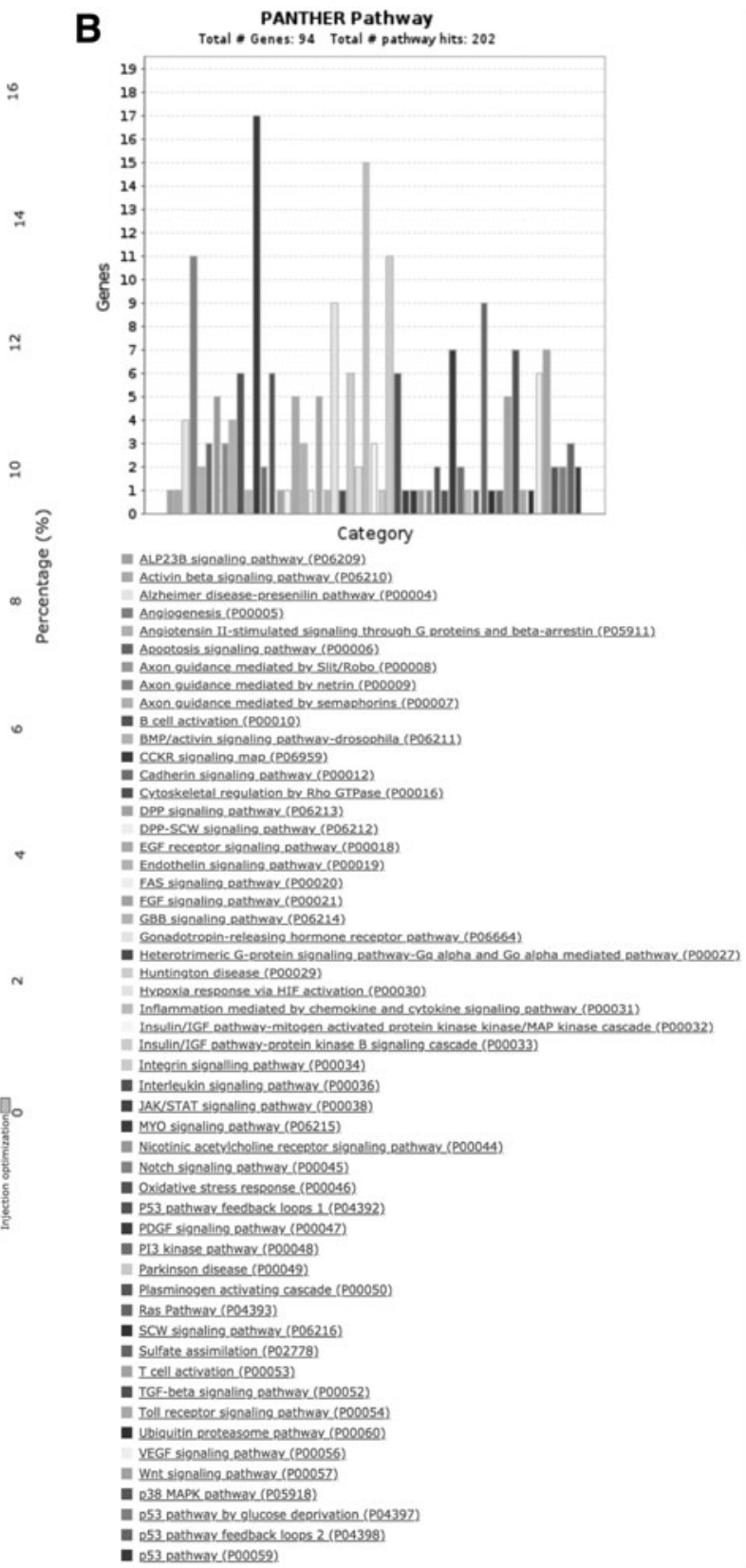

FIG. 4. Types of biological assessments performed on zebrafish xenograft models. (A) Biological analyses performed on zebrafish xenograft models through molecularly modified cell, PDX, and SC injections revealing major attributes studied in the field. (B) Representative bar chart of GO Panther pathway enrichment analysis on the modified genes revealing the more profoundly studied pathways. Figures are available in greater detail online. 
Transplantation of induced pluripotent stem cell (iPSC)driven differentiated cells, ${ }^{69}$ hematopoietic stem cells, ${ }^{70,71}$ and mesenchymal stem cells (from adipose tissue) ${ }^{72}$ are among those studied in zebrafish xenograft models. SC studies account for the $16.36 \%$ of all curated xenograft studies in ZenoFishDb v1.1 with incorporated details of the origin of SC and CSCs transplanted into zebrafish embryos (Fig. 3C and Supplementary Table S5).

\section{Biological assessments on zebrafish xenograft models}

Searches performed with ZenoFishDb v1.1 reveal a broad range of tumor-biology associated attributes in zebrafish xenograft studies, including tumor growth $(11.32 \%)$, proliferation $(10.61 \%)$, invasion $(9.67 \%)$, extravasation $(1.89 \%)$, migration $(7.55 \%)$, metastasis $(15.80 \%)$, angiogenesis $(8.73 \%)$, cytotoxicity $(0.94 \%)$, apoptosis $(1.42 \%)$, and drug sensitivity (1.18\%) (Fig. 4A and Supplementary Table S6). In addition, the list of modified genes (Supplementary Table S7) gathered from these articles has been subjected to an in-depth pathway analysis using GOPANTHER. ${ }^{73}$ The outcome of the pathway analysis (Fig. 4B) has revealed a total of 94 genes leading to 202 pathways out of which 18 major pathways are represented with at least 5 or more genes as visualized by the bar chart. Most frequently studied pathways include CCKR signaling, inflammation mediated by chemokine and cytokine signaling, integrin signaling, gonadotropin-releasing hormone receptor, angiogenesis, and Ras pathways (Fig. 4B).

In addition to these enriched pathways, we have also gathered information on the end point of biological assessments of each publication in our repertoire as hours postinjection (hpi) for embryos and as hpi or weeks postinjection (wpi) for adults. Forty-eight and $72 \mathrm{hpi}$ are among the most analyzed time points after injection, while other time points uniformly included are 24, 96, 120, and $144 \mathrm{hpi}$ in xenografted embryos (Supplementary Fig. S1).

Although not a drastic percentage difference has been detected in the majority of the end points, other parameters such as tissue of origin, cancer cell type, injected number of cells, or location could also affect the experimental course and selection of end time point. For instance, Mercatali et al., studied metastases of breast cancer cell lines of different invasive capacity of MCF7 (hormone receptor positive, noninvasive) and MDA-MB-231 (triple negative breast cancer, invasive) together with a patient-derived breast cancer bone metastasis primary cell line. Herein, at $120 \mathrm{hpi}$, only MDA-MB-231 cells and primary cells survived, disseminated, and colonized in other parts of the fish implying the importance of choice of cell line and type of assessments to be performed at a specific time point. ${ }^{58}$

Moshal et al. studied angiogenic capacity of human and mice lung tumor cell lines, H1299 (nonsmall cell lung carcinoma) and CL13 (lung adenocarcinoma), respectively. Both of these cell lines and a nontumorigenic 3T3-L1 cell line were injected to $\operatorname{Tg}(f l k 1: \mathrm{eGFP})$ fish at 24 hours postfertilization (hpf), and angiogenic capacity was assessed at $48 \mathrm{hpi}$ testing alkaline phosphatase activity. In addition, significant increases in the number and length of ectopic vessels were detected in tumorigenic cell lines confirming presence of angiogenesis at $48 \mathrm{hpi}^{74}$ Hence, when metastasis-related events such as extravasation, migration, invasion, and angiogenesis were considered together, a rel- atively homogenous distribution emerges for scoring xenografts at 48 or $72 \mathrm{hpi}$.

Based on data housed in ZenoFishDb v1.1, tumor growth and proliferation although generally not assessed solely are also collected frequently at 48 and $72 \mathrm{hpi}$. However, assessment-specific prolonged end points are also observed in xenotransplantation studies in embryos, for example, with respect to survival ${ }^{62}$ and immunohistochemical ${ }^{75}$ measurements. Xenotransplantation in adult fish on the other hand is scarce yet assessments are recorded by means of hpi, ${ }^{76,77}$ as well as wpi, ${ }^{28,78}$ onto our database (Supplementary Fig. S1 and Supplementary Table S8).

These findings altogether highlight the importance of variability in spatial and temporal characteristics of xenotransplantation studies that should be taken into consideration while addressing different biological assessments, as well as the choice of cell lines, PDXs, and injection sites. ZenoFishDb v1.1 allows for evaluation of such parameters readily helping users to plan and execute their experiments.

\section{Zebrafish xenograft model as a tool for drug screening}

Zebrafish has been long used for drug screening as thoroughly revived by different authors in the field. ${ }^{15,79}$ Yet, availability of zebrafish xenograft models further enhanced the applications of drug screening on human-derived tumor bearing fish. In fact, models such as ZeOncoTest have been used to refine and automate use of zebrafish xenotransplantation for cancer drug discovery. ${ }^{80}$ Using ZenoFishDb v1.1 one can identify individual studies harboring different routes of drug administration such as those given before transplantation, ${ }^{81-83}$ as well as those in which drugs are directly added to the fish water. ${ }^{84}$ More than 200 different drugs have been identified and incorporated into the current version of the database. Dasatinib, ${ }^{48,85}$ SU5416, ${ }^{17,86}$ and Doxorubicin $^{87}$ are among the most commonly used drugs, while use of nanoparticles ${ }^{88}$ and exosomes ${ }^{89}$ has been also recorded in the list of zebrafish xenograft drug studies (Supplementary Fig. S2 and Supplementary Table S9). Hence, ZenoFishDb v1.1 provides a platform for the feasible search, cataloging, and comparison of drug applications performed on zebrafish xenograft models.

\section{Zebrafish host modifications for xenotransplantation}

The availability of in vivo imaging of vascular development by $\mathrm{Tg}$ (flil:EGFP) zebrafish embryos ${ }^{90}$ provides great ease for visualization across embryonic development. In fact, a majority of the xenograft studies harboring angiogenesis, invasion, and metastasis assays ${ }^{49,91}$ benefits from $\operatorname{Tg}(f i 1: \mathrm{EGFP})$ line where the flil promoter, the earliestknown endothelial marker, ${ }^{92}$ is used for driving the green fluorescent protein (GFP) expression. Similarly, $\operatorname{Tg}(f k 1: \mathrm{EGFP})^{\mathrm{s} 843}$ zebrafish line ${ }^{93}$ generating green vasculature under $f k 1$ is widely used to investigate invasive and metastatic capacity of tumor cells. ${ }^{19,94}$ The use of transparent casper, as well as albino fish, has further improved visualization of transplanted cell behavior in zebrafish. ${ }^{95}$ Using ZenoFishDb v1.1, one can obtain a listing of all studies that contain zebrafish modified/mutant strains used with transplantation of cells with molecular modifications and PDX or SC xenografts. 
Other mutant and knockout/knockdown zebrafish strains are becoming central for understanding the effects of microenvironment in tumorigenesis. For instance, acetylcholinesterase mutant ache, harboring excess acetylcholine, is a model to test the role of ache deficiency of the host on size of the liver tumors. ${ }^{96}$ Similarly, cloche mutant fish is lacking nearly all blood cells and, therefore, functional circulation, and vasculature $\left(\right.$ cloche $\left.^{--}\right)$allows for testing whether metastasis and tumor growth require host vasculature. ${ }^{63,97}$ In addition, morpholinos (MO) that are widely applicable for discovery of gene function can be used in xenotransplantation to modify host microenvironment. For example, transplantation of retinoblastoma cells into zebrafish embryos microinjected with MO against vegf-aa lowered levels of metastasis compared to control MO-treated embryos. ${ }^{98}$ In another example, the injection of HCT116 cells into $\operatorname{Tg}$ (fil:EGFP) protein kinase D1 morphant abolished tumor angiogenesis. ${ }^{99}$

A search using ZenoFishDb v1.1 Visualization page, upon selecting the "host strain" column, shows the presence of transgenic $(55.74 \%)$, mutant $(20.08 \%)$, and/or morphant $(2.46 \%)$ strains used as modified host microenvironments
(Fig. 5A and Supplementary Table S10). Accordingly, a representative image of the subselected mutant "host details" and corresponding "host detail modifications" has been provided using the DataTable pages (Fig. 5B).

These studies demonstrate the undeniable power of using morphant, mutant, and transgenic zebrafish embryos and larvae to understand the role of microenvironment in human tumor growth, angiogenesis, and metastasis. ZenoFishDb v1.1 database thus can be useful in keeping up with the ever-increasing studies in the xenotransplantation field in which zebrafish host is often genetically and/or epigenetically modified.

\section{Zebrafish xenograft models from a technical point of view}

ZenoFishDb v1.1 can also be used to search the zebrafish literature for differences in the technical aspects of xenotransplantation, such as the site and timing of injection, number of cells injected, and types of tracking dyes used. Precise location of the injection site is crucial for the type of biological analysis to be performed in xenograft studies. In fact, yolk sac injections are ideally used for testing initiation

A

HOST MODIFICATIONS

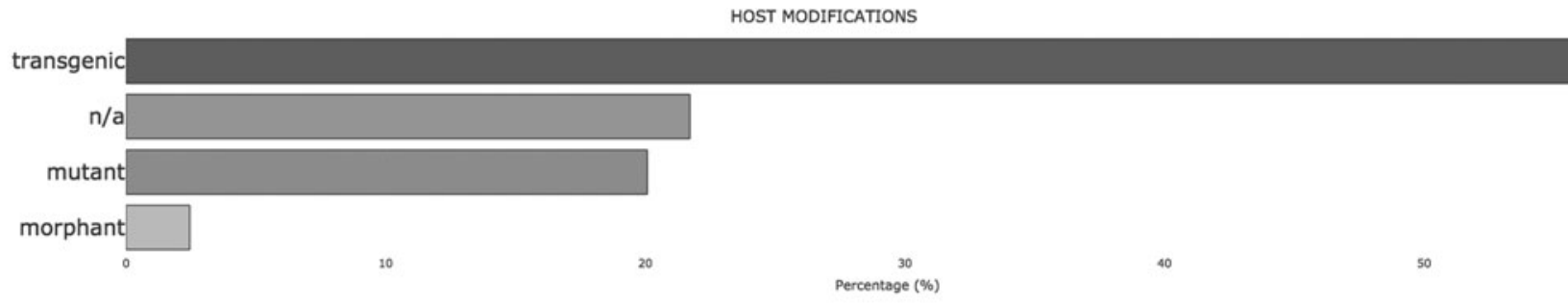

B

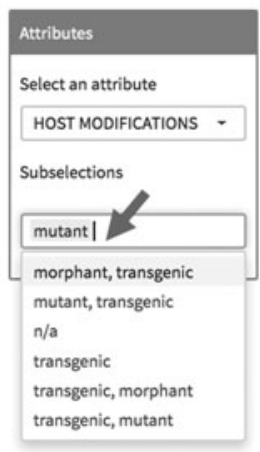

\begin{tabular}{|c|c|c|c|}
\hline \multicolumn{4}{|l|}{ Data Table } \\
\hline & & Search: [ & \\
\hline ZEBRAFISH LINES & $\begin{array}{l}\text { HOST } \\
\text { MODIFICATIONS }\end{array}$ & HOST MODIFICATIONS DETAILS & $\begin{array}{l}\text { CELL TRACKING } \\
\text { SOURCES }\end{array}$ \\
\hline Casper & mutant & Transparent embryo & GFP \\
\hline Casper & mutant & Transparent embryo & $\mathrm{n} / \mathrm{a}$ \\
\hline Casper & mutant & Transparent embryo & CMTPX-Red \\
\hline prkdc $/ /$; ;il2rga $/ \%$;casper & mutant & Transparent embryo, Lacks T, B and NK cells & GFP, CFSE, Dil \\
\hline Casper & mutant & Transparent embryo & CM-Dil \\
\hline Wild type, ache-sb55 mutant & mutant & achesb55 mutants (homorygous mutant: paralyzed at 3dpf,hererozygous mutant: normal development) & DiO, Dil, GFP \\
\hline Casper & mutant & Transparent embryo & GFP \\
\hline Casper & mutant & Casper (roy- $/-$; mitfa- $/-)$ & GFP \\
\hline Casper & mutant & Transparent embryo & CM-Dil \\
\hline Casper & mutant & Transparent embryo & GFP \\
\hline Casper & mutant & Transparent embryo & GFP \\
\hline Casper & mutant & Transparent embryo & CMTMR \\
\hline Wild type, Casper & mutant & Transparent embryo & GFP \\
\hline Casper & mutant & Transparent embryo & CM-Dil \\
\hline Casper & mutant & Transparent embryo & CM-Dil \\
\hline prkdc- $\%$, prkdc+\%- & mutant & Prkdc-Null severe combined immunodeficient (SCID) zebrafish & tRFP \\
\hline Casper & mutant & Transparent embryo & GFP, mCherry, CFSE \\
\hline Casper & mutant & Transparent embryo & CM-Dil, GFP \\
\hline
\end{tabular}

FIG. 5. ZenoFishDb v1.1 reveals distinct host modifications and microenvironment studies used in xenograft studies. (A) Graphical representation of host modifications obtained from the Visualization page. (B) Screenshot of DataTable page with "host modifications" attribute, and subselection choice of "mutant" attribute. Figures are available in greater detail online. 
A INJECTION SITE

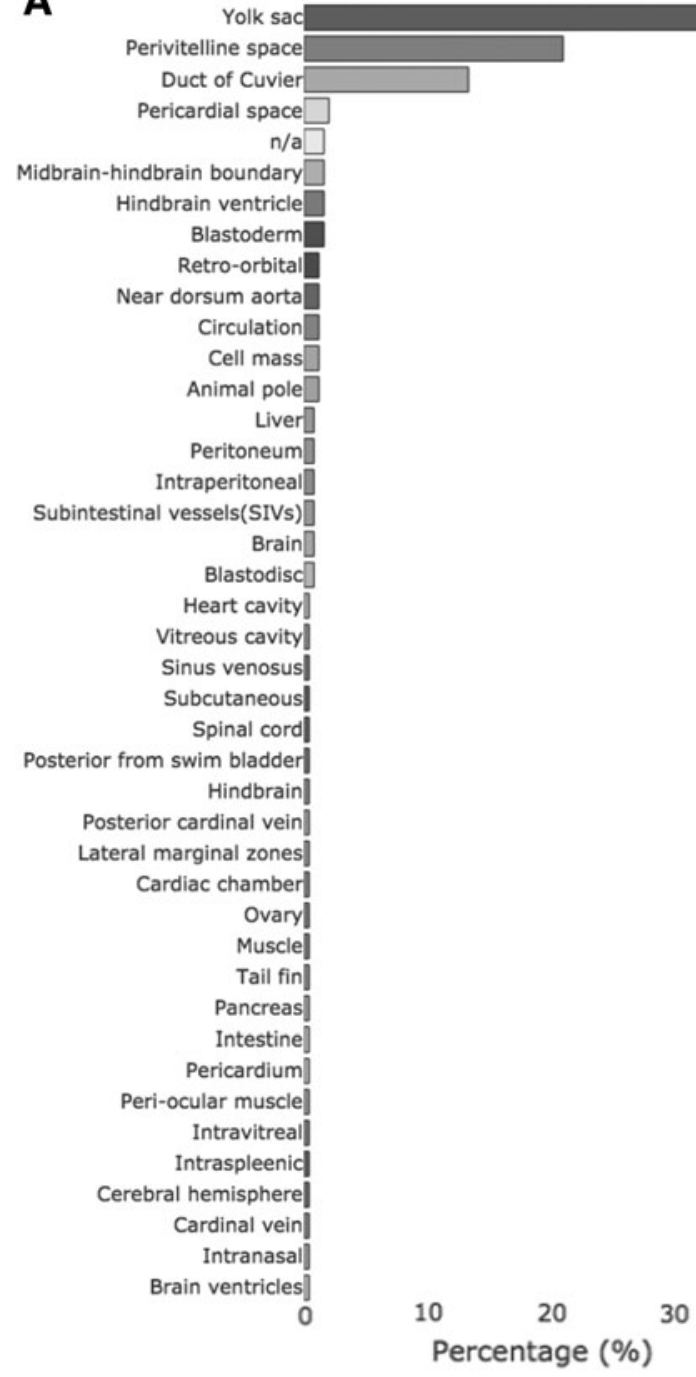

C

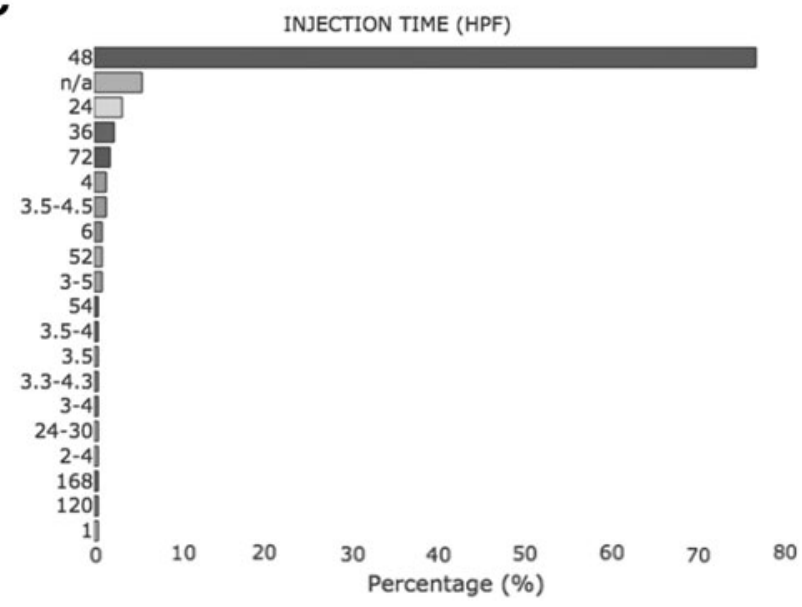

B

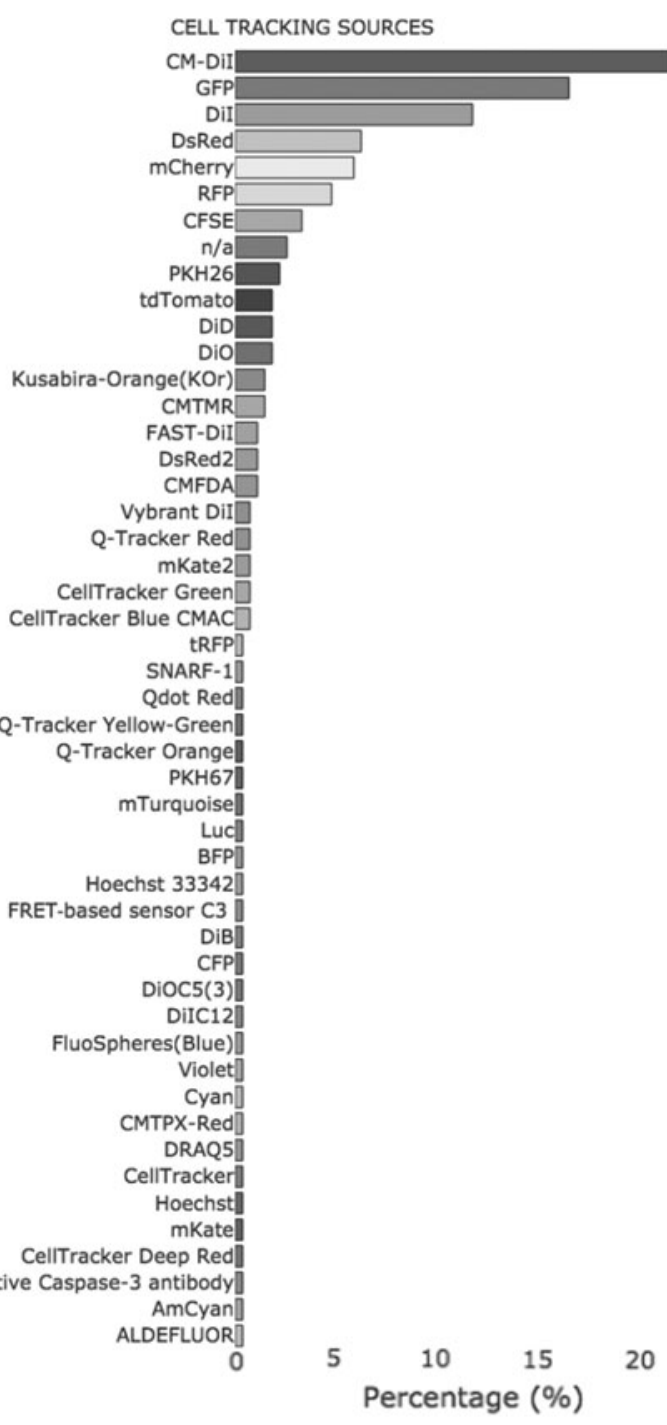

D

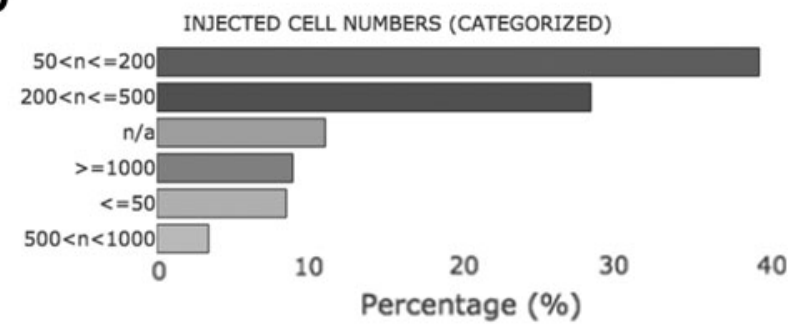

FIG. 6. ZenoFishDb v1.1 reveals statistical data on technical prospects of xenografting in zebrafish. (A) Injection sites; (B) cell tracking systems; (C) time of injection; (D) injected cell numbers-categorized. Figures are available in greater detail online. 
of tumor formation, tumor growth, or proliferation, ${ }^{39,40}$ whereas duct of Cuvier opens to the sinus venosus of the heart and allows analysis of circulating injected cells and hence cellular migration ${ }^{70}$ and metastasis to tail fin. ${ }^{100}$ Injection into the perivitelline space of the zebrafish embryo has been initially used for an angiogenesis assay by Nicoli and Pre$\mathrm{sta}^{101}$ and similarly by other groups where the ectopic SIVsprouting has been tested. ${ }^{99}$ Although these exemplify common examples of injection sites for specific biological assessments, there are other possibilities. Statistical representation of injection sites using ZenoFishDb v1.1 reveals the yolk sac $(37.10 \%)$ as the most preferred injection site followed by perivitelline space $(20.97 \%)$ and duct of Cuvier (13.31\%) (Fig. 6A and Supplementary Table S11).

Transparent zebrafish embryos are enabling precise tracking of the location and migration of the fluorescently labeled transplanted cells. In fact, solid tumors inside the yolk ${ }^{96,102,103}$ or brain ${ }^{104-107}$ and migrating cells in the veins ${ }^{103,108}$ can be detected readily by fluorescence microscopy. Cell lines transplanted in zebrafish are often stained by fluorescent protein vectors such as GFP, ${ }^{29}$ mCherry, ${ }^{100}$ DsRed, ${ }^{106}$ and live dyes, among which visualization by CMDiI, ${ }^{103}$ DiI, ${ }^{96,102} \mathrm{DiD}^{109}{ }^{\mathrm{CFSE}},{ }^{110}$ and $\mathrm{CMTMR}^{37}$ is the most frequently used based on a ZenoFishDb v1.1 search (Fig. 6B and Supplementary Table S12).

Another technical aspect highlights the timing of the injection to be performed at different injection points in zebrafish embryos $(92.66 \%)$ and/or adult fish $(6.42 \%)$, which holds great importance for the strategic decision-making for assessments to be performed. ${ }^{111}$ Great majority of the embryos $(76.85 \%)$ have undergone the injection during the first 48 hpf (Fig. 6C and Supplementary Table S13).

In addition, we have also reviewed the differences in the number of cells injected. In the literature, studies testing different number of cells in different biological concepts exist; among these Fior et al., ${ }^{59}$ for example, injected 500 and 1000 colorectal cancer primary cells into the perivitelline space for testing early and late metastatic events, respectively. However, another study assessing tumor size used 50100 cells for cell lines and 500 cells for patient samples when injecting into the yolk sac. ${ }^{60}$ Using ZenoFishDb v1.1, we show the percentage of studies with different number of cells injected, for example, $50<n \leq 200$ cells (39.57\%) or $200<n \leq 500$ cells $(28.51 \%)$, where $n$ represents the number of cells. Injections harboring cells $n \leq 50,500<n<1000$, and $n \geq 1000$ also exist, yet they are sparser (Fig. 6D and Supplementary Table S14). ZenoFishDb v1.1, hence, covers technical aspects of zebrafish xenografting models pinpointing the specifics of experimental design.

\section{Conclusions and Future Perspectives}

ZenoFishDb v1.1 offers an easy access to zebrafish xenograft studies with a specific focus on PDXs and the molecular modifications in the transplanted cells, as well as on host microenvironment. In addition, our findings address recent and novel perspectives in the literature, such as use of SCs and CSCs, along with therapeutic approaches that can be useful in translational medicine. Future inclusions of zebrafish xenotransplantation studies that use unmodified cells or hosts and drug screens with different time intervals and dosing are also planned in the upcoming versions of Zeno-
FishDb v1.1. Moreover, keywords used for searching literature will be diversified and generalized to be more comprehensive in case "xenograft" or "xenotransplant" is not included in the study abstract. In conclusion, ZenoFishDb v1.1 incorporates a thorough and systematic review of 211 transplantation studies highlighting the extent of xenografting molecularly modified cells in wild-type/transgenic/ knockout/morphant/mutant zebrafish (reviewed until November 29, 2019) and shows that the emerging applications of in vivo cancer and personalized medicine in the zebrafish xenograft field complement the studies performed in mice and other organisms.

\section{Authors' Contributions}

O.K. conceptualized the ZenoFishDb v1.1 and supervised the study; S.T., O.K., M.E.A. identified criteria to be curated; S.T. curated the data and drafted the data table; T.K. developed and tested the database; S.T., M.E.A., D.G., A.G.K. performed literature search; S.T., O.K., and M.E.A. wrote the article, and S.T. made the figures; M.E.A., D.G., A.G.K., T.K. helped with data curation; and all authors tested the database and read, revised, and authorized the article.

\section{Acknowledgments}

The authors thank Bilkent University Graduate School for providing scholarships to S.T., T.K., D.G., and A.G.K. and the anonymous reviewers for their invaluable comments.

\section{Disclosure Statement}

No competing financial interests exist.

\section{Funding Information}

No funding was received for this article.

\section{Supplementary Material}

Supplementary Figure S1 Supplementary Figure S2 Supplementary Table S1 Supplementary Table S2 Supplementary Table S3 Supplementary Table S4 Supplementary Table S5 Supplementary Table S6 Supplementary Table S7 Supplementary Table S8 Supplementary Table S9 Supplementary Table S10 Supplementary Table S11 Supplementary Table S12 Supplementary Table S13 Supplementary Table S14

\section{References}

1. Bosma GC, Custer RP, Bosma MJ. A severe combined immunodeficiency mutation in the mouse. Nature 1983; 301:527-530.

2. Clemmesen J. On transplantation of tumor cells to normal and pre-irradiated heterologous organisms. Am Assoc Cancer Res J 1937;29:313-332. 
3. Giovanella BC, Yim SO, Stehlin JS, Williams LJ Jr. Development of invasive tumors in the "nude" mouse after injection of cultured human melanoma cells. J Natl Cancer Inst 1972;48:1531-1533.

4. Rygaard J, Povlsen CO. Heterotransplantation of a human malignant tumour to "Nude" mice. Acta Pathol Microbiol Scand 1969;77:758-760.

5. Day CP, Merlino G, Van Dyke T. Preclinical mouse cancer models: a maze of opportunities and challenges. Cell 2015;163:39-53.

6. Richmond A, Su Y. Mouse xenograft models vs GEM models for human cancer therapeutics. Dis Model Mech 2008; 1:78-82.

7. Bult CJ, Krupke DM, Begley DA, Richardson JE, Neuhauser SB, Sundberg JP, et al. Mouse Tumor Biology (MTB): a database of mouse models for human cancer. Nucleic Acids Res 2015;43:D818-D824.

8. Conway T, Wazny J, Bromage A, Tymms M, Sooraj D, Williams ED, et al. Xenome-a tool for classifying reads from xenograft samples. Bioinformatics 2012;28:1172i178.

9. He S, Hu B, Li C, Lin P, Tang WG, Sun YF, et al. PDXliver: a database of liver cancer patient derived xenograft mouse models. BMC Cancer 2018;18:550.

10. Kluin RJC, Kemper K, Kuilman T, de Ruiter JR, Iyer V, Forment JV, et al. XenofilteR: computational deconvolution of mouse and human reads in tumor xenograft sequence data. BMC Bioinformatics 2018;19:366.

11. Conte N, Mason JC, Halmagyi C, Neuhauser S, Mosaku A, Yordanova G, et al. PDX Finder: a portal for patientderived tumor xenograft model discovery. Nucleic Acids Res 2019;47:D1073-D1079.

12. Taconic Biosciences: Genetically Engineered Rodent Models. Taconic Biosciences, Inc. 2020. https://www.taconic .com/resources/xenograft-cell-line-reference-database/

13. Lee LM, Seftor EA, Bonde G, Cornell RA, Hendrix MJ. The fate of human malignant melanoma cells transplanted into zebrafish embryos: assessment of migration and cell division in the absence of tumor formation. Dev Dyn 2005;233:1560-1570.

14. Zhang B, Xuan C, Ji Y, Zhang W, Wang D. Zebrafish xenotransplantation as a tool for in vivo cancer study. Fam Cancer 2015; 14:487-493.

15. Brown HK, Schiavone K, Tazzyman S, Heymann D, Chico TJ. Zebrafish xenograft models of cancer and metastasis for drug discovery. Expert Opin Drug Discov 2017;12:379-389.

16. Drabsch Y, Snaar-Jagalska BE, Ten Dijke P. Fish tales: The use of zebrafish xenograft human cancer cell models. Histol Histopathol 2017;32:673-686.

17. Zhao C, Wang X, Zhao Y, Li Z, Lin S, Wei Y, et al. A novel xenograft model in zebrafish for high-resolution investigating dynamics of neovascularization in tumors. PLoS One 2011;6:e21768.

18. Xiong P, Xiao LY, Yang R, Guo Q, Zhao YQ, Li W, et al. Flotillin-1 promotes cell growth and metastasis in oral squamous cell carcinoma. Neoplasma 2013;60:395-405.

19. Thomas C, Rajapaksa G, Nikolos F, Hao R, Katchy A, McCollum CW, et al. ERbeta1 represses basal breast cancer epithelial to mesenchymal transition by destabilizing EGFR. Breast Cancer Res 2012;14:R148.

20. Halasz M, Polgar B, Berta G, Czimbalek L, SzekeresBartho J. Progesterone-induced blocking factor differentially regulates trophoblast and tumor invasion by altering matrix metalloproteinase activity. Cell Mol Life Sci 2013; 70:4617-4630.

21. Herrera J, Beisang DJ, Peterson M, Forster C, Gilbertsen A, Benyumov A, et al. Dicer1 deficiency in the idiopathic pulmonary fibrosis fibroblastic focus promotes fibrosis by suppressing microRNA biogenesis. Am J Respir Crit Care Med 2018;198:486-496.

22. Chang W, Cheng J, Allaire J, Xie Y, McPherson J: shiny: Web Application Framework for R., 2018. https://shiny .rstudio.com/

23. R Core Team: R: A Language and Environment for Statistical Computing. R Foundation for Statistical Computing, Vienna, Austria, 2013.

24. Xie Y, Cheng J, Tan X: DT: A Wrapper of the JavaScript Library 'DataTables'. 2018. https://github.com/rstudio/DT

25. Wickham H, François R, Henry L, Müller K: dplyr: A Grammar of Data Manipulation. R Package Version 0.7.8., 2018.

26. Sievert C: Plotly for R. 2018. https://plotly.com/r/

27. Bansal N, Davis S, Tereshchenko I, Budak-Alpdogan T, Zhong H, Stein MN, et al. Enrichment of human prostate cancer cells with tumor initiating properties in mouse and zebrafish xenografts by differential adhesion. Prostate 2014;74:187-200.

28. Jung IH, Chung YY, Jung DE, Kim YJ, Kim do H, Kim $\mathrm{KS}$, et al. Impaired lymphocytes development and xenotransplantation of gastrointestinal tumor cells in PrkdcNull SCID zebrafish model. Neoplasia 2016;18:468-479.

29. Martinez-Ordonez A, Seoane S, Cabezas P, Eiro N, Sendon-Lago J, Macia M, et al. Breast cancer metastasis to liver and lung is facilitated by Pit-1-CXCL12-CXCR4 axis. Oncogene 2018;37:1430-1444.

30. Wang H, Schaefer T, Konantz M, Braun M, Varga Z, Paczulla AM, et al. Prominent oncogenic roles of EVI1 in breast carcinoma. Cancer Res 2017;77:2148-2160.

31. Wu PC, Lu JW, Yang JY, Lin IH, Ou DL, Lin YH, et al. H3K9 histone methyltransferase, KMT1E/SETDB1, cooperates with the SMAD2/3 pathway to suppress lung cancer metastasis. Cancer Res 2014;74:7333-7343.

32. Chiavacci E, Rizzo M, Pitto L, Patella F, Evangelista M, Mariani L, et al. The zebrafish/tumor xenograft angiogenesis assay as a tool for screening anti-angiogenic miRNAs. Cytotechnology 2015;67:969-975.

33. Chen C, Choudhury S, Wangsa D, Lescott CJ, Wilkins DJ, Sripadhan P, et al. A multiplex preclinical model for adenoid cystic carcinoma of the salivary gland identifies regorafenib as a potential therapeutic drug. Sci Rep 2017;7:11410.

34. Lin J, Zhang W, Zhao JJ, Kwart AH, Yang C, Ma D, et al. A clinically relevant in vivo zebrafish model of human multiple myeloma to study preclinical therapeutic efficacy. Blood 2016;128:249-252.

35. Wu JQ, Zhai J, Li CY, Tan AM, Wei P, Shen LZ, et al. Patient-derived xenograft in zebrafish embryos: a new platform for translational research in gastric cancer. J Exp Clin Cancer Res 2017;36:160.

36. Othman N, Nagoor NH. miR-608 regulates apoptosis in human lung adenocarcinoma via regulation of AKT2. Int $\mathrm{J}$ Oncol 2017;51:1757-1764.

37. Cichon MA, Szentpetery Z, Caley MP, Papadakis ES, Mackenzie IC, Brennan $\mathrm{CH}$, et al. The receptor tyrosine kinase Axl regulates cell-cell adhesion and stemness in cutaneous squamous cell carcinoma. Oncogene 2014;33: 4185-4192.

38. Schaefer T, Wang H, Mir P, Konantz M, Pereboom TC, Paczulla AM, et al. Molecular and functional interactions 
between AKT and SOX2 in breast carcinoma. Oncotarget 2015;6:43540-43556.

39. Huang CY, Lee CH, Tu CC, Wu CH, Huang MT, Wei PL, et al. Glucose-regulated protein 94 mediates progression and metastasis of esophageal squamous cell carcinoma via mitochondrial function and the NF-kB/COX-2/VEGF axis. Oncotarget 2018;9:9425-9441.

40. Corkery DP, Clarke LE, Gebremeskel S, Salsman J, Pinder $\mathrm{J}$, Le Page C, et al. Loss of PRP4K drives anoikis resistance in part by dysregulation of epidermal growth factor receptor endosomal trafficking. Oncogene 2018;37:174-184.

41. Chapman A, Fernandez del Ama L, Ferguson J, Kamarashev J, Wellbrock C, Hurlstone A. Heterogeneous tumor subpopulations cooperate to drive invasion. Cell Rep 2014;8:688-695.

42. de Boeck M, Cui C, Mulder AA, Jost CR, Ikeno S, Ten Dijke P. Smad6 determines BMP-regulated invasive behaviour of breast cancer cells in a zebrafish xenograft model. Sci Rep 2016;6:24968.

43. Britto DD, Wyroba B, Chen W, Lockwood RA, Tran KB, Shepherd PR, et al. Macrophages enhance Vegfa-driven angiogenesis in an embryonic zebrafish tumour xenograft model. Dis Model Mech 2018;11:dmm035998.

44. Weijts B, Westendorp B, Hien BT, Martinez-Lopez LM, Zijp M, Thurlings I, et al. Atypical E2Fs inhibit tumor angiogenesis. Oncogene 2018;37:271-276.

45. He S, Lamers GE, Beenakker JW, Cui C, Ghotra VP, Danen EH, et al. Neutrophil-mediated experimental metastasis is enhanced by VEGFR inhibition in a zebrafish xenograft model. J Pathol 2012;227:431-445.

46. Smith MP, Ferguson J, Arozarena I, Hayward R, Marais $\mathrm{R}$, Chapman A, et al. Effect of SMURF2 targeting on susceptibility to MEK inhibitors in melanoma. J Natl Cancer Inst 2013;105:33-46.

47. Cho SJ, Kook MC, Lee JH, Shin JY, Park J, Bae YK, et al. Peroxisome proliferator-activated receptor gamma upregulates galectin- 9 and predicts prognosis in intestinal-type gastric cancer. Int J Cancer 2015;136:810-820.

48. von Massenhausen A, Sanders C, Bragelmann J, Konantz M, Queisser A, Vogel W, et al. Targeting DDR2 in head and neck squamous cell carcinoma with dasatinib. Int $\mathbf{J}$ Cancer 2016;139:2359-2369.

49. Li Y, Drabsch Y, Pujuguet P, Ren J, van Laar T, Zhang L, et al. Genetic depletion and pharmacological targeting of alphav integrin in breast cancer cells impairs metastasis in zebrafish and mouse xenograft models. Breast Cancer Res 2015; $17: 28$

50. Gaytan-Cervantes J, Gonzalez-Torres C, Maldonado V, Zampedri C, Ceballos-Cancino G, Melendez-Zajgla J. Protein Sam68 regulates the alternative splicing of survivin DEx3. J Biol Chem 2017;292:13745-13757.

51. Simbulan-Rosenthal CM, Dougherty R, Vakili S, Ferraro AM, Kuo LW, Alobaidi R, et al. CRISPR-Cas9 knockdown and induced expression of CD133 reveal essential roles in melanoma invasion and metastasis. Cancers (Basel) 2019;11:1490.

52. Jung J, Seol HS, Chang S. The generation and application of patient-derived xenograft model for cancer research. Cancer Res Treat 2018;50:1-10.

53. Mitra A, Mishra L, Li S. Technologies for deriving primary tumor cells for use in personalized cancer therapy. Trends Biotechnol 2013;31:347-354.

54. Cassidy JW, Caldas C, Bruna A. Maintaining tumor heterogeneity in patient-derived tumor xenografts. Cancer Res 2015;75:2963-2968.
55. Barriuso J, Nagaraju R, Hurlstone A. Zebrafish: a new companion for translational research in oncology. Clin Cancer Res 2015;21:969-975.

56. Collins AT, Lang SH. A systematic review of the validity of patient derived xenograft (PDX) models: the implications for translational research and personalised medicine. PeerJ 2018;6:e5981.

57. Eden CJ, Ju B, Murugesan M, Phoenix TN, Nimmervoll $\mathrm{B}$, Tong Y, et al. Orthotopic models of pediatric brain tumors in zebrafish. Oncogene 2015;34:1736-1742.

58. Mercatali L, La Manna F, Groenewoud A, Casadei R, Recine F, Miserocchi G, et al. Development of a patientderived xenograft (PDX) of breast cancer bone metastasis in a zebrafish model. Int J Mol Sci 2016;17:1375.

59. Fior R, Povoa V, Mendes RV, Carvalho T, Gomes A, Figueiredo N, et al. Single-cell functional and chemosensitive profiling of combinatorial colorectal therapy in zebrafish xenografts. Proc Natl Acad Sci U S A 2017;114: E8234-E8243.

60. Bentley VL, Veinotte CJ, Corkery DP, Pinder JB, LeBlanc MA, Bedard K, et al. Focused chemical genomics using zebrafish xenotransplantation as a pre-clinical therapeutic platform for T-cell acute lymphoblastic leukemia. Haematologica 2015;100:70-76.

61. Gaudenzi G, Albertelli M, Dicitore A, Wurth R, Gatto F, Barbieri F, et al. Patient-derived xenograft in zebrafish embryos: a new platform for translational research in neuroendocrine tumors. Endocrine 2017;57:214-219.

62. Banasavadi-Siddegowda YK, Welker AM, An M, Yang $\mathrm{X}$, Zhou W, Shi G, et al. PRMT5 as a druggable target for glioblastoma therapy. Neuro Oncol 2018;20:753-763.

63. Marques IJ, Weiss FU, Vlecken DH, Nitsche C, Bakkers J, Lagendijk AK, et al. Metastatic behaviour of primary human tumours in a zebrafish xenotransplantation model. BMC Cancer 2009;9:128.

64. Bonnet D, Dick JE. Human acute myeloid leukemia is organized as a hierarchy that originates from a primitive hematopoietic cell. Nat Med 1997;3:730-737.

65. Baiocchi M, Biffoni M, Ricci-Vitiani L, Pilozzi E, De Maria R. New models for cancer research: human cancer stem cell xenografts. Curr Opin Pharmacol 2010;10:380384.

66. Chen L, Groenewoud A, Tulotta C, Zoni E, Kruithof-de Julio M, van der Horst $\mathrm{G}$, et al. A zebrafish xenograft model for studying human cancer stem cells in distant metastasis and therapy response. Methods Cell Biol 2017; 138:471-496.

67. Eguiara A, Holgado O, Beloqui I, Abalde L, Sanchez Y, Callol C, et al. Xenografts in zebrafish embryos as a rapid functional assay for breast cancer stem-like cell identification. Cell Cycle 2011;10:3751-3757.

68. Zhang B, Shimada Y, Kuroyanagi J, Ariyoshi M, Nomoto $\mathrm{T}$, Shintou $\mathrm{T}$, et al. In vivo selective imaging and inhibition of leukemia stem-like cells using the fluorescent carbocyanine derivative, DiOC5(3). Biomaterials 2015; $52: 14-25$.

69. Chan XY, Black R, Dickerman K, Federico J, Levesque $\mathrm{M}$, Mumm J, et al. Three-dimensional vascular network assembly from diabetic patient-derived induced pluripotent stem cells. Arterioscler Thromb Vasc Biol 2015;35: 2677-2685.

70. Staal FJ, Spaink HP, Fibbe WE. Visualizing human hematopoietic stem cell trafficking in vivo using a zebrafish xenograft model. Stem Cells Dev 2016;25:360-365. 
71. Hamilton N, Sabroe I, Renshaw SA. A method for transplantation of human HSCs into zebrafish, to replace humanised murine transplantation models. F1000Res 2018;7:594.

72. Han HW, Hsu SH. Chitosan-hyaluronan based 3D coculture platform for studying the crosstalk of lung cancer cells and mesenchymal stem cells. Acta Biomater 2016; 42:157-167.

73. Mi H, Dong Q, Muruganujan A, Gaudet P, Lewis S, Thomas PD. PANTHER version 7: improved phylogenetic trees, orthologs and collaboration with the Gene Ontology Consortium. Nucleic Acids Res 2010;38:D204D210.

74. Moshal KS, Ferri-Lagneau KF, Haider J, Pardhanani P, Leung T. Discriminating different cancer cells using a zebrafish in vivo assay. Cancers (Basel) 2011;3:4102-4113.

75. Welker AM, Jaros BD, An M, Beattie CE. Changes in tumor cell heterogeneity after chemotherapy treatment in a xenograft model of glioblastoma. Neuroscience 2017; 356:35-43.

76. Hayes MN, McCarthy K, Jin A, Oliveira ML, Iyer S, Garcia SP, et al. Vangl2/RhoA signaling pathway regulates stem cell self-renewal programs and growth in rhabdomyosarcoma. Cell Stem Cell 2018;22:414-427.e6.

77. Khan N, Mahajan NK, Sinha P, Jayandharan GR. An efficient method to generate xenograft tumor models of acute myeloid leukemia and hepatocellular carcinoma in adult zebrafish. Blood Cells Mol Dis 2019;75:48-55.

78. Yan C, Brunson DC, Tang Q, Do D, Iftimia NA, Moore $\mathrm{JC}$, et al. Visualizing engrafted human cancer and therapy responses in immunodeficient zebrafish. Cell 2019;177: 1903-1914.e14.

79. MacRae CA, Peterson RT. Zebrafish as tools for drug discovery. Nat Rev Drug Discov 2015;14:721-731.

80. Cornet C, Dyballa S, Terriente J, Di Giacomo V. ZeOncoTest: refining and automating the zebrafish xenograft model for drug discovery in cancer. Pharmaceuticals (Basel) 2019;13:1.

81. Vazquez Rodriguez G, Abrahamsson A, Jensen LDE, Dabrosin C. Adipocytes promote early steps of breast cancer cell dissemination via interleukin-8. Front Immunol 2018;9:1767.

82. Vazquez Rodriguez G, Abrahamsson A, Jensen LD, Dabrosin C. Estradiol promotes breast cancer cell migration via recruitment and activation of neutrophils. Cancer Immunol Res 2017;5:234-247.

83. Marranci A, D’Aurizio R, Vencken S, Mero S, Guzzolino E, Rizzo M, et al. Systematic evaluation of the microRNAome through miR-CATCHv2.0 identifies positive and negative regulators of BRAF-X1 mRNA. RNA Biol 2019;16:865-878.

84. van der Ent W, Burrello C, Teunisse AF, Ksander BR, van der Velden PA, Jager MJ, et al. Modeling of human uveal melanoma in zebrafish xenograft embryos. Invest Ophthalmol Vis Sci 2014;55:6612-6622.

85. Timmermans-Sprang EPM, Mestemaker HM, Steenlage RR, Mol JA. Dasatinib inhibition of cSRC prevents the migration and metastasis of canine mammary cancer cells with enhanced Wnt and HER signalling. Vet Comp Oncol 2019;17:413-426.

86. Lin QH, Qu W, Xu J, Feng F, He MF. 1-Methoxycarbonybeta-carboline from Picrasma quassioides exerts antiangiogenic properties in HUVECs in vitro and zebrafish embryos in vivo. Chin J Nat Med 2018;16:599-609.
87. Fu A, Peh YM, Ngan W, Wei N, Luo KQ. Rapid identification of antimicrometastases drugs using integrated model systems with two dimensional monolayer, three dimensional spheroids, and zebrafish xenotransplantation tumors. Biotechnol Bioeng 2018;115:2828-2843.

88. Harfouche R, Basu S, Soni S, Hentschel DM, Mashelkar RA, Sengupta S. Nanoparticle-mediated targeting of phosphatidylinositol-3-kinase signaling inhibits angiogenesis. Angiogenesis 2009;12:325-338.

89. Shtam T, Naryzhny S, Samsonov R, Karasik D, Mizgirev I, Kopylov A, et al. Plasma exosomes stimulate breast cancer metastasis through surface interactions and activation of FAK signaling. Breast Cancer Res Treat 2019; 174:129-141.

90. Lawson ND, Weinstein BM. In vivo imaging of embryonic vascular development using transgenic zebrafish. Dev Biol 2002;248:307-318.

91. Drabsch Y, He S, Zhang L, Snaar-Jagalska BE, Ten Dijke P. Transforming growth factor-beta signalling controls human breast cancer metastasis in a zebrafish xenograft model. Breast Cancer Res 2013;15:R106.

92. Thompson MA, Ransom DG, Pratt SJ, MacLennan H, Kieran MW, Detrich HW 3rd, et al. The cloche and spadetail genes differentially affect hematopoiesis and vasculogenesis. Dev Biol 1998;197:248-269.

93. Jin SW, Beis D, Mitchell T, Chen JN, Stainier DY. Cellular and molecular analyses of vascular tube and lumen formation in zebrafish. Development 2005;132:51995209.

94. Wei J, Zhang J, Si Y, Kanada M, Zhang Z, Terakawa S, et al. Blockage of LMP1-modulated store-operated $\mathrm{Ca}(2+)$ entry reduces metastatic potential in nasopharyngeal carcinoma cell. Cancer Lett 2015;360:234-244.

95. Corkery DP, Dellaire G, Berman JN. Leukaemia xenotransplantation in zebrafish-chemotherapy response assay in vivo. Br J Haematol 2011;153:786-789.

96. Avci ME, Keskus AG, Targen S, Isilak ME, Ozturk M, Atalay RC, et al. Development of a novel zebrafish xenograft model in ache mutants using liver cancer cell lines. Sci Rep 2018;8:1570.

97. Zhao C, Zhang W, Zhao Y, Yang Y, Luo H, Ji G, et al. Endothelial cords promote tumor initial growth prior to vascular function through a paracrine mechanism. Sci Rep 2016;6:19404.

98. Chen X, Wang J, Cao Z, Hosaka K, Jensen L, Yang H, et al. Invasiveness and metastasis of retinoblastoma in an orthotopic zebrafish tumor model. Sci Rep 2015;5:10351.

99. Hollenbach M, Stoll SJ, Jorgens K, Seufferlein T, Kroll J. Different regulation of physiological and tumor angiogenesis in zebrafish by protein kinase D1 (PKD1). PLoS One 2013;8:e68033.

100. Spender LC, Ferguson GJ, Liu S, Cui C, Girotti MR, Sibbet G, et al. Mutational activation of BRAF confers sensitivity to transforming growth factor beta inhibitors in human cancer cells. Oncotarget 2016;7:81995-82012.

101. Nicoli S, Presta M. The zebrafish/tumor xenograft angiogenesis assay. Nat Protoc 2007;2:2918-2923.

102. Gabellini C, Gomez-Abenza E, Ibanez-Molero S, Tupone MG, Perez-Oliva AB, de Oliveira S, et al. Interleukin 8 mediates bcl-xL-induced enhancement of human melanoma cell dissemination and angiogenesis in a zebrafish xenograft model. Int J Cancer 2018;142:584-596.

103. Ghotra VP, He S, de Bont H, van der Ent W, Spaink HP, van de Water B, et al. Automated whole animal bio- 
imaging assay for human cancer dissemination. PLoS One 2012;7:e31281.

104. Hamilton L, Astell KR, Velikova G, Sieger D. A zebrafish live imaging model reveals differential responses of microglia toward glioblastoma cells in vivo. Zebrafish 2016; 13:523-534.

105. Wehmas LC, Tanguay RL, Punnoose A, Greenwood JA. Developing a novel embryo-larval zebrafish xenograft assay to prioritize human glioblastoma therapeutics. Zebrafish 2016;13:317-329.

106. Vittori M, Breznik B, Hrovat K, Kenig S, Lah TT. RECQ1 helicase silencing decreases the tumour growth rate of U87 glioblastoma cell xenografts in zebrafish embryos. Genes (Basel) 2017;8:222.

107. Rampazzo E, Persano L, Pistollato F, Moro E, Frasson C, Porazzi $\mathrm{P}$, et al. Wnt activation promotes neuronal differentiation of glioblastoma. Cell Death Dis 2013;4:e500.

108. Cao Y, Hoeppner LH, Bach S, E G, Guo Y, Wang E, et al. Neuropilin-2 promotes extravasation and metastasis by interacting with endothelial alpha5 integrin. Cancer Res 2013;73:4579-4590.
109. Tan DS, Haaland B, Gan JM, Tham SC, Sinha I, Tan EH, et al. Bosutinib inhibits migration and invasion via ACK1 in KRAS mutant non-small cell lung cancer. Mol Cancer 2014;13:13.

110. Chiang KC, Hsu SY, Lin SJ, Yeh CN, Pang JH, Wang SY, et al. PTEN insufficiency Increases breast cancer cell metastasis in vitro and in vivo in a xenograft zebrafish model. Anticancer Res 2016;36:3997-4005.

111. Yan C, Yang Q, Do D, Brunson DC, Langenau DM. Adult immune compromised zebrafish for xenograft cell transplantation studies. EBioMedicine 2019;47:24-26.

Address correspondence to: Ozlen Копи, PhD Department of Molecular Biology and Genetics Bilkent University Ankara 06800 Turkey

E-mail: konu@fen.bilkent.edu.tr 